\title{
铁氢配合物对一级酰胺脱水成腈的催化作用
}

\author{
郑婷婷 $a, b$ 王洋洋 $a, c$ 杨在孝 ${ }^{a}$ 孙宏建 ${ }^{a}$ 李晓燕 $*, a$ \\ $\left({ }^{a}\right.$ 山东大学化学与化工学院 教育部特种功能聚集体材料重点实验室 济南 250100) \\ ${ }^{b}$ 首都师范大学化学系 北京 100048) \\ ( ${ }^{c}$ 西北师范大学化学化工学院 兰州 730070)
}

\begin{abstract}
摘要 主要研究了苯硒酚基铁氢配合物 $c i s-\left[(\mathrm{ArSe}) \mathrm{FeH}\left(\mathrm{PMe}_{3}\right)_{4}\right]\left[\mathrm{Ar}=\mathrm{Ph}(\mathbf{1}), p-\mathrm{MeOC}_{6} \mathrm{H}_{4}\right.$ (2) 和o- $\mathrm{MeC}_{6} \mathrm{H}_{4}$ (3)]对一级酰胺 脱水成腈的催化作用. 实验证明当选用 $(\mathrm{EtO})_{3} \mathrm{SiH}$ 为还原剂时, 在较为温和的条件下这三种配合物均对一级酰胺脱水为 腈的过程都具有良好的催化作用. 该催化体系对于芳基酰胺中苯环上的取代基具有很好的耐受性. 与斥电性基团相比, 吸电性基团对该催化反应比较有利.
\end{abstract}

关键词 铁氢配合物; 硒酚; 硒配位; 酰胺脱水; 腈制备

\section{Catalytic Effect of Iron Hydrides on Dehydration of Primary Amides to Nitriles}

\author{
Zheng, Tingting ${ }^{a, b} \quad$ Wang, Yangyang ${ }^{a, c} \quad$ Yang, Zaixiao $^{a}$ \\ Sun, Hongjian $^{a} \quad$ Li, Xiaoyan ${ }^{*, a}$ \\ $\left({ }^{a}\right.$ Key Laboratory of Special Functional Aggregated Materials, Ministry of Education, School of Chemistry and \\ Chemical Engineering, Shandong University, Jinan 250100) \\ $\left({ }^{b}\right.$ Department of Chemistry, Capital Normal University, Beijing 100048) \\ ( ${ }^{c}$ College of Chemistry and Chemical Engineering, Northwest Normal University, Lanzhou 730070)
}

\begin{abstract}
Three cis-selenophenolato iron hydrides $\left[c i s-\left[(\mathrm{ArSe}) \mathrm{FeH}\left(\mathrm{PMe}_{3}\right)_{4}\right]\left(\mathrm{Ar}=\mathrm{Ph}(\mathbf{1}), p-\mathrm{MeOC}_{6} \mathrm{H}_{4}(\mathbf{2})\right.\right.$ and $o-\mathrm{MeC}_{6} \mathrm{H}_{4}$ (3)], were used as catalysts for the dehydration of primary amides to nitriles. The experimental results show that three complexes have good catalytic effect on reductive dehydration of primary amide to nitrile under mild conditions using $(\mathrm{EtO})_{3} \mathrm{SiH}$ as reducing agent. The catalytic system is well tolerated to the substituents on the benzene ring in aromatic amides. Compared with electron-donating group, electron-withdrawing group is more advantageous to the catalytic reaction.

Keywords iron hydride; selenophenol; Se-coordination; reductive amide dehydration; nitrile preparation
\end{abstract}

腈在有机合成化学中是一类十分重要的中间体 ${ }^{[1,2]}$. 利用一级酰胺脱水来制备腈是一种十分有效的方法 ${ }^{[3,4]}$. 而传统上脱水利用的是强脱水剂, 如 $\mathrm{SOCl}_{2}{ }^{[5]}$ 和 $\mathrm{P}_{2} \mathrm{O}_{5}{ }^{[6]}$ 等. 该过程不仅为化学计量比的反应, 而且会产生大量 的废液. 因此, 该方法并非一个环境友好的方法. 为了 克服这一缺点, 人们发展了一些催化剂来催化一级酰胺 脱水转化为腈的过程. 这方面较早的一些工作主要采用 贵金属催化剂, 如 $\mathrm{Rh}^{[7]}, \mathrm{Ru}^{[8]}, \mathrm{Ir}^{[9]}$ 和 $\mathrm{Pt}^{[10]}$. 贵金属不仅 价格昂贵, 而且有一定的毒性. 因此, 近年来一些廉价 金属的配合物已被应用于一级酰胺向腈的转化反应 ${ }^{[11]}$.
实验已证明二烷基锡的氧化物在中性条件下可催化一 级酰胺转化为腈 ${ }^{[12]}$. 氯化锌在乙腈的水溶液中也可催 化该过程 ${ }^{[13]}$. Weidauer 等 ${ }^{[14]}$ 报道了铜对一级酰胺脱水过 程的催化作用. 铁几乎没有毒性, 而且是地壳中含量最 丰富的过渡金属, 价格便宜. 因此, 铁配合物被认为是 最理想的金属催化剂. Enthaler ${ }^{[15]}$ 发现铁亦可以催化一 级酰胺转化为腈的反应. 在聚甲基氢硅氧烷(PMHS)存 在的条件下铁的 $\mathrm{N}$-杂环卡宾 (NHC)配合物可使苯甲酰 胺转化为苯甲 腈 ${ }^{[16]}$. 铁的环戊二烯基 NHC 配合物也可 利用苯基氢硅烷催化苯甲酰胺脱水生成苯甲腈 ${ }^{[17]}$. 我

* Corresponding author. E-mail: xli63@sdu.edu.cn

Received March 30, 2019; revised June 3, 2019; published online July 2, 2019.

Project supported by the National Natural Science Foundation of China (No. 21372143).

国家自然科学基金(No. 21372143)资助项目. 
们课题组 ${ }^{[11,18 ~ 27]}$ 围绕惰性键的活化和新型金属有机化 学物尤其是金属有机氢化物的合成及催化活性研究做 了大量研究工作. 最近, 我们 ${ }^{[11]}$ 报道了 $[\mathrm{CNC}]$-钳式钴 (III)氢配合物催化一级酰胺脱水为腈的反应, (EtO) $)_{3} \mathrm{SiH}$ 是该过程的还原剂. 综上所述, 廉价金属催化的一级酰 胺转化为腈的反应已经取得了一些进展, 但在催化剂的 制备、还原剂的选择和底物取代基团的耐受性等方面还 存在一些问题，该领域的工作仍需加强.

2016 年我们 ${ }^{[18]}$ 曾发表了三甲基膦支持的苯硫酚基 的铁氢配合物对于一级酰胺还原为腈的催化作用. 2018 年我们 ${ }^{[19]}$ 还证明三甲基膦支持的苯硒酚基铁氢配合物 1 3 是醛酮硅氢化反应的有效催化剂(图 1). 为了进一 步比较硫硒同系物的化学性质和催化作用规律性, 本工 作探讨了苯硒酚基的铁氢配合物 $1 \sim 3$ 对一级酰胺脱水 转化为腈的催化作用活性.

\section{1 结果与讨论}

为了寻找合适的反应条件, 以对氯苯甲酰胺为底物 进行了初步实验(表 1). 以 $2 \mathrm{~mol} \%$ 的 $\mathrm{Fe}\left(\mathrm{PMe}_{3}\right)_{4}$ 作为催化 剂, 3 equiv. 三乙氧基硅烷在四氢呋喃(THF)溶液中 60
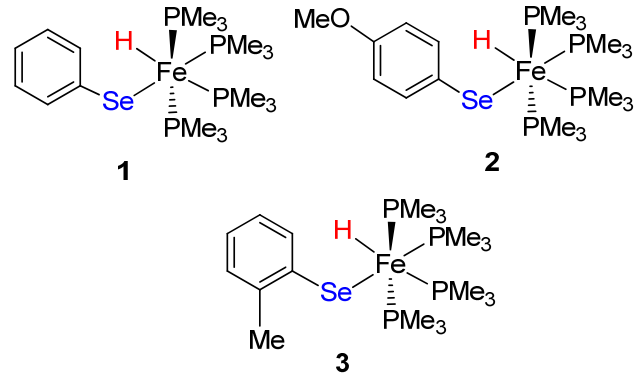

图 1 铁(II)氢配合物 $1 \sim 3$ 的结构

Figure 1 Structures of hydrido iron(II) complexes $\mathbf{1} \sim \mathbf{3}$

${ }^{\circ} \mathrm{C}$ 下反应 $24 \mathrm{~h}$, 无产物生成(Entry 1, 表 1). 当利用苯硒 酚基铁氢配合物 $\mathbf{1} \sim \mathbf{3}$ 作为催化剂进行反应时, 转化率 有显著的提升. 其中, 配合物 1 的催化活性最高, 以 99\%的转化率得到对氯苯甲腈(Entry 4, 表 1). 配合物 2 和 3 只有 $77 \%$ 和 $54 \%$ 的中等收率(Entries 2, 3, 表 1). 这 些实验结果说明这三种苯硒酚基铁氢配合物的催化活 性顺序为 $1>\mathbf{2}>\mathbf{3}$. 这个顺序与我们原来报道的苯硫酚 基铁氢配合物对一级酰胺脱水为腈的催化活性顺序一 致 ${ }^{[18]}$. 说明配合物催化剂苯环上斥电性基团的引入会

表 1 催化最优条件的節选 ${ }^{a}$

Table 1 Exploration of conditions for amide dehydration

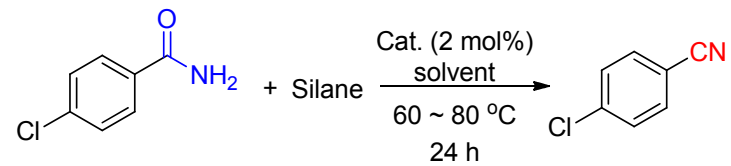

\begin{tabular}{|c|c|c|c|c|c|c|c|}
\hline Entry & Catalyst & Loading/mol\% & Silane & Solvent & $T /{ }^{\circ} \mathrm{C}$ & $t / \mathrm{h}$ & Conv. $^{b} / \%$ \\
\hline 1 & $\mathrm{Fe}\left(\mathrm{PMe}_{3}\right)_{4}$ & 2 & $(\mathrm{EtO})_{3} \mathrm{SiH}$ & THF & 60 & 24 & 0 \\
\hline 2 & 2 & 2 & $(\mathrm{EtO})_{3} \mathrm{SiH}$ & THF & 60 & 24 & 77 \\
\hline 3 & 3 & 2 & $(\mathrm{EtO})_{3} \mathrm{SiH}$ & THF & 60 & 24 & 54 \\
\hline 4 & 1 & 2 & $(\mathrm{EtO})_{3} \mathrm{SiH}$ & THF & 60 & 24 & 99 \\
\hline 5 & 1 & 1 & $(\mathrm{EtO})_{3} \mathrm{SiH}$ & THF & 60 & 24 & 82 \\
\hline 6 & 1 & 2 & $\mathrm{Et}_{3} \mathrm{SiH}$ & THF & 60 & 24 & 23 \\
\hline 7 & 1 & 2 & $\mathrm{PhSiH}_{3}$ & THF & 60 & 24 & 30 \\
\hline 8 & 1 & 2 & $\mathrm{Ph}_{2} \mathrm{SiH}_{2}$ & THF & 60 & 24 & 61 \\
\hline 9 & 1 & 2 & $\mathrm{Ph}_{3} \mathrm{SiH}$ & THF & 60 & 24 & 0 \\
\hline 10 & 1 & 2 & TMDS & THF & 60 & 24 & 28 \\
\hline 11 & 1 & 2 & PMHS & THF & 60 & 24 & 0 \\
\hline 12 & 1 & 2 & $(\mathrm{EtO})_{3} \mathrm{SiH}$ & Toluene & 60 & 24 & 87 \\
\hline 13 & 1 & 2 & $(\mathrm{EtO})_{3} \mathrm{SiH}$ & Pentane & 60 & 24 & 25 \\
\hline 14 & 1 & 2 & $(\mathrm{EtO})_{3} \mathrm{SiH}$ & DMF & 60 & 24 & 46 \\
\hline 15 & 1 & 2 & $(\mathrm{EtO})_{3} \mathrm{SiH}$ & DMSO & 60 & 24 & 79 \\
\hline 16 & 1 & 2 & $(\mathrm{EtO})_{3} \mathrm{SiH}$ & Dioxane & 60 & 24 & 85 \\
\hline 17 & 1 & 2 & $(\mathrm{EtO})_{3} \mathrm{SiH}$ & $\mathrm{CH}_{3} \mathrm{CN}$ & 60 & 24 & 63 \\
\hline 18 & 1 & 2 & $(\mathrm{EtO})_{3} \mathrm{SiH}$ & THF & 50 & 24 & 90 \\
\hline 19 & 1 & 2 & $(\mathrm{EtO})_{3} \mathrm{SiH}$ & THF & 55 & 24 & 97 \\
\hline 20 & 1 & 2 & $(\mathrm{EtO})_{3} \mathrm{SiH}$ & THF & 60 & 13 & 58 \\
\hline 21 & 1 & 2 & $(\mathrm{EtO})_{3} \mathrm{SiH}$ & THF & 60 & 16 & 78 \\
\hline 22 & 1 & 2 & $(\mathrm{EtO})_{3} \mathrm{SiH}$ & THF & 60 & 20 & 94 \\
\hline
\end{tabular}

${ }^{a}$ Catalytic reaction conditions: amide $(1.0 \mathrm{mmol})$, hydrosilane $(3.0 \mathrm{mmol})$ and cat. in $2 \mathrm{~mL}$ of solvent. ${ }^{b}$ Determined by $\mathrm{GC}$ analysis. 
降低其催化活性. 推测可能由于苯环上甲基和甲氧基的 给电子效应导致铁中心富电子, 从而使 $\mathrm{Fe}-\mathrm{H}$ 键难以断 裂, 降低其催化活性. 对于催化剂 3, 由于苯环上的甲 基处于邻位, 空间位阻也可能是一个影响催化活性的因 素. 因此, 选用配合物 $\mathbf{1}$ 作为最佳的催化剂, 对催化剂 的量、硅烷种类、溶剂、反应的温度和时间进行一系列 的优化. 当将催化剂的量由 $2 \mathrm{~mol} \%$ 降低为 $1 \mathrm{~mol} \%$ 时, 反应转化率降低至 $82 \%$ (Entry 5, 表 1). 在对硅烷的篮 选中发现, 当使用三乙基硅烷、苯基硅烷、二苯基硅烷 和四甲基二硅氮烷(TMDS)作为还原剂时, 转化率均较 低(Entries 6 8,10, 表 1), 而在使用三苯基硅烷和聚甲 基氢硅氧烷(PMHS)作为还原剂时, 催化反应则无法进 行(Entries 9, 11, 表 1). 这可能由于这些硅烷的空间位 阻较大, 不利于催化反应的进行. 除四氢呋喃作为溶剂 外, 还测试了甲苯、正戊烷、 $N, N$-二甲基甲酰胺(DMF)、 二甲基亚砜(DMSO)、1,4-二噁烷(Dioxane)和乙腈, 发现 反应效果都不理想(Entries 12 17, 表 1). 降低反应的 温度或者缩短反应的时间, 对于催化反应都是不利的 (Entries 19 23, 表 1). 最终, 确定反应的最佳条件为: 2 $\mathrm{mol} \%$ 的铁氢配合物 $\mathbf{1}$ 作为催化剂, 3 equiv.三乙氧基硅烷 作为还原剂, 四氢呋喃作为反应溶剂, $60{ }^{\circ} \mathrm{C}$ 下反应 24 h.

在上述优化的条件下, 拓展了酰胺脱水的底物范围 (表 2). 对于苯甲酰胺来说, 以 $75 \%$ 的产率得到苯甲腈. 首先, 研究了空间效应和电子效应对酰胺脱水反应的影 响. 当底物酰胺的苯环上对位带有基团时, 产率普遍较 高, 而注意到当邻位或者间位带有取代基时, 产率降低. 相比较给电子基团, 当苯环上带有吸电子基团时, 反应 效果较好, 产率可达 $77 \% \sim 90 \%$. 此外, 具有挑战性的 杂环族伯酰胺 2-吡啶酰胺也以优异的产率转化为相应 的腈类化合物 (79\%). 值得注意的是, 对于可还原的官 能团, 例如硝基和烯烃, 在 $2 \mathrm{~mol} \%$ 的配合物 $\mathbf{1}$ 催化剂的 条件下, 将酰胺脱水为腈具有高效的选择性. 反应中只 选择性地将酰胺基还原为腈, 而对其它基团进行了保 留. 最后, 对烷基酰胺(芐基酰胺和对羟基芐基酰胺)进 行测试, 发现也能以中等的产率脱水为腈(74\%和 $85 \%$ ). 我们认为该催化过程的机理可能与 Buchwald 提出的关 于铜氢配合物催化酰胺脱水成腈的机理是类似的 ${ }^{[28]}$.

\section{2 结论}

开发了一种利用三乙氧基硅烷为还原剂, 苯硒酚基 铁氢化合物为催化剂, 催化伯酰胺脱水为腈的新方法. 催化剂的制备方法简单, 反应条件温和. 该催化体系和 催化条件对于芳基酰胺中苯环上的取代基具有很好的 耐受性. 吸电性基团比斥电性基团对催化反应更有利.
表 2 配合物 $\mathbf{1}$ 催化的酰胺脱水为腈的研究 ${ }^{a}$

Table 2 Study on dehydration of amides to nitriles catalyzed by 1

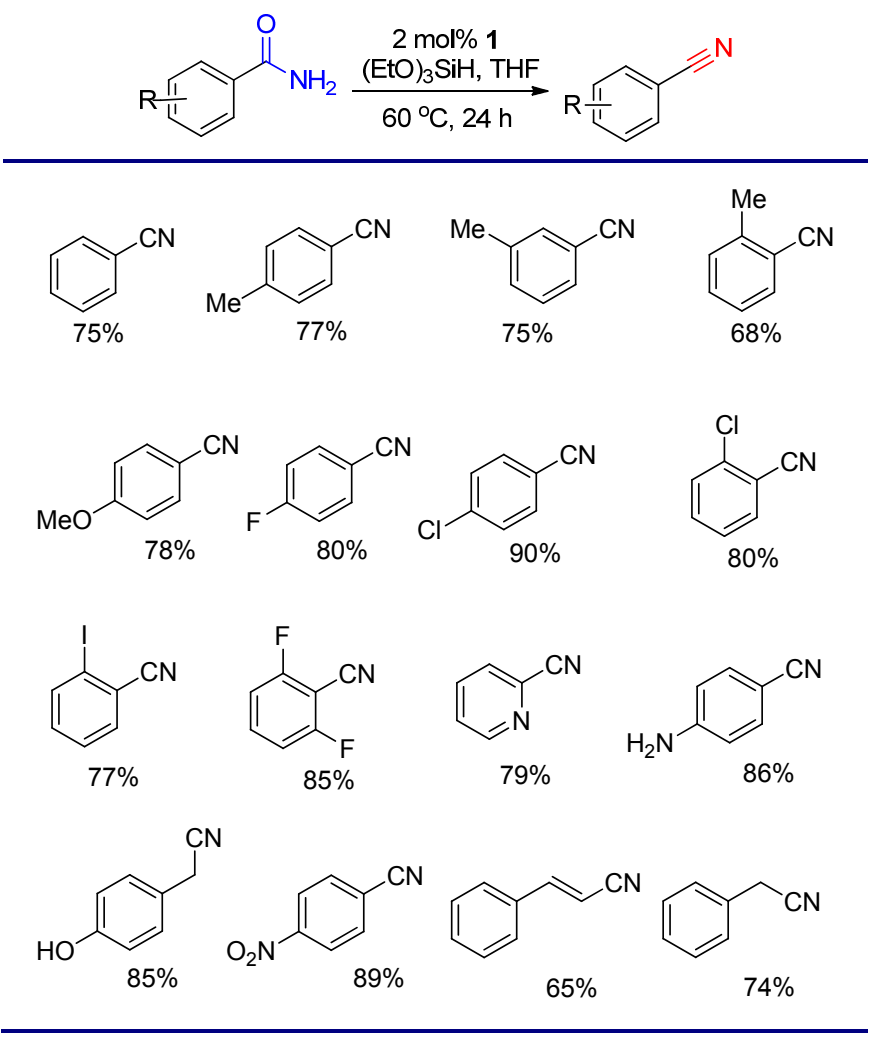

${ }^{a}$ Catalytic reaction conditions: amide $(1.0 \mathrm{mmol}),(\mathrm{EtO})_{3} \mathrm{SiH}(3.0 \mathrm{mmol})$ and $\mathbf{1}$ $(0.02 \mathrm{mmol})$ in $2 \mathrm{~mL}$ of THF, $60{ }^{\circ} \mathrm{C}, 24 \mathrm{~h}$; isolated yields.

取代基的空间位阻对于实验结果有一定的影响. 与属于 同系物的苯硫酚基铁氢配合物催化一级酰胺脱水为腈 的实验结果相比较 ${ }^{[18]}$, 两个催化体系实验结果的规律 和趋势是相似的. 只是苯硒酚基铁氢配合物的催化活性 稍微低了一点. 本工作还说明苯硒酚基铁氢配合物不仅 可以有效地催化醛酮硅氢化反应 ${ }^{[19]}$, 而且还可应用于 一级酰胺脱水为腈的过程.

\section{3 实验部分}

\section{1 仪器与试剂}

使用标准的真空技术制备和处理对空气敏感的化 合物. 化合物的红外光谱采用石蜡油液膜的方式获得, 红外光谱仪为 Bruker ALPHA FT-IR. 化合物的核磁共振 谱图在 Bruker Avance $300 \mathrm{MHz}$ 仪器上测得. 所用气相 色谱仪为 Fuli 9790. 所有的底物酰胺在购买后未经纯化 直接使用. 苯硒酚基铁氢配合物 $1 \sim 3$ 是利用苯硒酚与 $\mathrm{Fe}\left(\mathrm{PMe}_{3}\right)_{4}$ 的反应参考文献[19]制备的.

\section{2 铁氢配合物对酰胺脱水反应的催化作用}

在室温下氮气氛围中, 将底物酰胺 $(1.0 \mathrm{mmol})$ 、 $(\mathrm{EtO})_{3} \mathrm{SiH}(3.0 \mathrm{mmol}) 、$ 催化剂(2 $\left.\mathrm{mol} \%\right)$ 和溶剂四氢呋喃 (THF) (2 mL)加入 $25 \mathrm{~mL}$ 的 Schlenk 管中. 将反应混合物 
在 $60{ }^{\circ} \mathrm{C}$ 下搅拌反应 $24 \mathrm{~h}$. 反应过程中用气相色谱 $(\mathrm{GC})$ 和薄层色谱(TLC)检测反应进程. 待反应结束后, 用石 油醚 $(10 \mathrm{~mL} \times 3)$ 萃取产物, 合并有机相, 用无水 $\mathrm{Na}_{2} \mathrm{SO}_{4}$ 干燥. 减压旋蒸除去有机溶剂, 粗产品使用凝胶柱色谱 法进一步纯化.

苯甲腈 ${ }^{[11,30]}$ : 无色液体. ${ }^{1} \mathrm{H} \mathrm{NMR}\left(300 \mathrm{MHz}, \mathrm{CDCl}_{3}\right)$ $\delta: 7.43 \sim 7.31(\mathrm{~m}, 3 \mathrm{H}), 7.28 \sim 7.20(\mathrm{~m}, 2 \mathrm{H}) ;{ }^{13} \mathrm{C}$ NMR $(75$ $\mathrm{MHz}, \mathrm{CDCl}_{3}, 298$ K) $\delta: 132.7,131.9,129.1,118.6,112.2$.

对甲基苯甲腈 ${ }^{[11,30]}$ : 白色晶体. m.p. $27 \sim 29{ }^{\circ} \mathrm{C}$ (文 献值 $\left.{ }^{[31]}: 29 \sim 30{ }^{\circ} \mathrm{C}\right) ;{ }^{1} \mathrm{H} \mathrm{NMR}\left(300 \mathrm{MHz}, \mathrm{CDCl}_{3}\right) \delta: 7.54$ (d, $J=9 \mathrm{~Hz}, 2 \mathrm{H}), 7.27$ (d, $J=6 \mathrm{~Hz}, 2 \mathrm{H}), 2.42$ (s, Me, 3H); ${ }^{13} \mathrm{C}$ NMR (75 MHz, $\left.\mathrm{CDCl}_{3}, 298 \mathrm{~K}\right) \delta: 142.9,131.3,129.0$, 118.4, 108.5, 21.0.

间甲基苯甲腈 ${ }^{[11]}$ : 无色液体. ${ }^{1} \mathrm{H}$ NMR $(300 \mathrm{MHz}$, $\left.\mathrm{CDCl}_{3}\right) \delta: 7.27 \sim 7.14(\mathrm{~m}, 4 \mathrm{H}), 2.19$ (s, Me, 3H); ${ }^{13} \mathrm{C} \mathrm{NMR}$ $\left(75 \mathrm{MHz}, \mathrm{CDCl}_{3}, 298 \mathrm{~K}\right) \delta: 139.2,133.6,132.2,129.0$, $128.9,118.8,112.0,21.3$.

邻甲基苯甲腈 ${ }^{[11]}$ : 无色液体. ${ }^{1} \mathrm{H}$ NMR $(300 \mathrm{MHz}$, $\left.\mathrm{CDCl}_{3}\right) \delta: 7.48 \sim 7.35(\mathrm{~m}, 2 \mathrm{H}), 7.22 \sim 7.13(\mathrm{~m}, 2 \mathrm{H}), 2.43$ $(\mathrm{s}, \mathrm{Me}, 3 \mathrm{H}) ;{ }^{13} \mathrm{C} \mathrm{NMR}\left(75 \mathrm{MHz}, \mathrm{CDCl}_{3}, 298 \mathrm{~K}\right) \delta: 141.8$, 132.6, 132.4, 130.2, 126.2, 118.1, 112.7, 20.4.

对甲氧基苯甲腈 ${ }^{[11,30]}$ : 白色固体. m.p. $58 \sim 59{ }^{\circ} \mathrm{C}$ (文献值 ${ }^{[31]}: 58 \sim 59{ }^{\circ} \mathrm{C}$ ); ${ }^{1} \mathrm{H}$ NMR $\left(300 \mathrm{MHz}, \mathrm{CDCl}_{3}\right) \delta$ : 7.52 (d, $J=9 \mathrm{~Hz}, 2 \mathrm{H}), 6.88$ (d, $J=9 \mathrm{~Hz}, 2 \mathrm{H}), 3.79$ (s, OMe, $3 \mathrm{H}) ;{ }^{13} \mathrm{C}$ NMR (75 MHz, $\left.\mathrm{CDCl}_{3}, 298 \mathrm{~K}\right) \delta: 162.8$, 134.0, 119.2, 114.7, 104.0, 55.5.

对氟苯甲腈 ${ }^{[11,30]}$ : 白色固体. ${ }^{1} \mathrm{H}$ NMR $(300 \mathrm{MHz}$, $\left.\mathrm{CDCl}_{3}\right) \delta: 7.64 \sim 7.58(\mathrm{~m}, 2 \mathrm{H}), 7.14 \sim 7.07(\mathrm{~m}, 2 \mathrm{H}) ;{ }^{13} \mathrm{C}$ NMR (75 MHz, $\left.\mathrm{CDCl}_{3}, 298 \mathrm{~K}\right) \delta: 165.0(\mathrm{~d}, J=256.5 \mathrm{~Hz})$, 134.6, 118.6, 116.8 (d, $J=22.8 \mathrm{~Hz}), 108.6$.

对氯苯甲腈 ${ }^{[11,30]}$ : 白色固体, m.p. 92 93 ${ }^{\circ} \mathrm{C}$ (文献 值 $\left.{ }^{[31]}: 91 \sim 92{ }^{\circ} \mathrm{C}\right) ;{ }^{1} \mathrm{H}$ NMR $\left(300 \mathrm{MHz}, \mathrm{CDCl}_{3}\right) \delta: 7.56 \sim$ $7.48(\mathrm{~m}, 2 \mathrm{H}), 7.42 \sim 7.36(\mathrm{~m}, 2 \mathrm{H}) ;{ }^{13} \mathrm{C} \mathrm{NMR}(75 \mathrm{MHz}$, $\left.\mathrm{CDCl}_{3}, 298 \mathrm{~K}\right) \delta$ : 138.6, 132.6, 128.6, 116.9, 110.0 .

邻氯苯甲腈 ${ }^{[11,30]}$ : 针状晶体. m.p. $44 \sim 45{ }^{\circ} \mathrm{C}$ (文献 值 $\left.{ }^{[32]}: 44 \sim 46{ }^{\circ} \mathrm{C}\right) ;{ }^{1} \mathrm{H}$ NMR $\left(300 \mathrm{MHz}, \mathrm{CDCl}_{3}\right) \delta: 7.67 \sim$ $7.19(\mathrm{~m}, 4 \mathrm{H}) ;{ }^{13} \mathrm{C} \mathrm{NMR}\left(75 \mathrm{MHz}, \mathrm{CDCl}_{3}, 298 \mathrm{~K}\right) \delta: 136.8$, 134.0, 133.9, 130.1, 127.2, 115.9, 113.3.

邻碘苯甲腈 ${ }^{[11,30]}$ : 白色固体. ${ }^{1} \mathrm{H}$ NMR (300 MHz, $\left.\mathrm{CDCl}_{3}\right) \delta: 7.84 \sim 7.81(\mathrm{~m}, 1 \mathrm{H}), 7.53 \sim 7.50(\mathrm{~m}, 1 \mathrm{H})$, $7.42 \sim 7.37(\mathrm{~m}, 1 \mathrm{H}), 7.25 \sim 7.19(\mathrm{~m}, 1 \mathrm{H}) ;{ }^{13} \mathrm{C}$ NMR $(75$ $\left.\mathrm{MHz}, \mathrm{CDCl}_{3}, 298 \mathrm{~K}\right) \delta: 139.5,134.3,133.9,128.5,120.4$, $119.4,98.5$.

2,6-二氟苯甲腈 ${ }^{[11,30]}$ : 白色固体. ${ }^{1} \mathrm{H}$ NMR (300 $\left.\mathrm{MHz}, \mathrm{CDCl}_{3}\right) \delta: 7.61 \sim 7.47(\mathrm{~m}, 1 \mathrm{H}), 6.98(\mathrm{t}, J=9 \mathrm{~Hz}$,
$2 \mathrm{H}) ;{ }^{13} \mathrm{C}$ NMR (75 MHz, $\left.\mathrm{CDCl}_{3}, 298 \mathrm{~K}\right) \delta: 163.3(\mathrm{~d}, J=$ 260.0, 4.5 Hz), 135.6 (t, $J=10.1 \mathrm{~Hz}$ ), 112.2 (dd, $J=19.4$, $3.8 \mathrm{~Hz}), 109.1,92.6(\mathrm{t}, J=19.1 \mathrm{~Hz})$.

2-吡啶甲腈 ${ }^{[11,30]}$ : 白色固体, m.p. $25 \sim 26{ }^{\circ} \mathrm{C}$ (文献 值 $\left.{ }^{[33]}: 24 \sim 25{ }^{\circ} \mathrm{C}\right) ;{ }^{1} \mathrm{H}$ NMR $\left(300 \mathrm{MHz}, \mathrm{CDCl}_{3}\right) \delta: 8.68$ (d, $J=3 \mathrm{~Hz}, 1 \mathrm{H}), 7.84 \sim 7.78(\mathrm{~m}, 1 \mathrm{H}), 7.68 \sim 7.64(\mathrm{~m}, 1 \mathrm{H})$, $7.52 \sim 7.47(\mathrm{~m}, 1 \mathrm{H}) ;{ }^{13} \mathrm{C} \mathrm{NMR}\left(75 \mathrm{MHz}, \mathrm{CDCl}_{3}, 298 \mathrm{~K}\right) \delta$ : 150.4, 136.4, 133.3, 127.8, 126.3, 116.3.

对氨基苯甲腈 ${ }^{[11,30]}$ : 白色固体，m.p. $85 \sim 86{ }^{\circ} \mathrm{C}$ (文 献值 $\left.{ }^{[34]}: 84 \sim 85{ }^{\circ} \mathrm{C}\right) ; \quad{ }^{1} \mathrm{H}$ NMR $\left(300 \mathrm{MHz}^{\circ} \mathrm{CDCl}_{3}\right) \delta$ : $7.34 \sim 7.19(\mathrm{~m}, 2 \mathrm{H}), 6.56 \sim 6.52(\mathrm{~m}, 2 \mathrm{H}), 4.30\left(\mathrm{~s}, \mathrm{NH}_{2}\right.$, $2 \mathrm{H}) ;{ }^{13} \mathrm{C} \mathrm{NMR}\left(75 \mathrm{MHz}, \mathrm{CDCl}_{3}, 298 \mathrm{~K}\right) \delta: 151.0,133.7$, 120.6, 114.4, 99.3.

对羟基苯乙腈 ${ }^{[11,30]}$ : 白色固体, m.p. $65 \sim 69{ }^{\circ} \mathrm{C}$ (文 献值 $\left.{ }^{[35]}: 65 \sim 70\right) ;{ }^{1} \mathrm{H}$ NMR (300 MHz, $\left.\mathrm{CDCl}_{3}\right) \delta: 7.04(\mathrm{~d}$, $J=9 \mathrm{~Hz}, 2 \mathrm{H}), 6.74(\mathrm{~d}, J=6 \mathrm{~Hz}, 2 \mathrm{H}), 6.34$ (s, OH, 1H), $3.56\left(\mathrm{~s}, \mathrm{CH}_{2}, 2 \mathrm{H}\right) ;{ }^{13} \mathrm{C} \mathrm{NMR}\left(75 \mathrm{MHz}, \mathrm{CDCl}_{3}, 298 \mathrm{~K}\right) \delta$ : 155.8, 129.3, 121.3, 118.6, 116.2, 22.8 .

对硝基苯甲腈 ${ }^{[11,30]}$ : 白色固体，m.p. 149 $150{ }^{\circ} \mathrm{C}$ (文献值 ${ }^{[31]}: 149 \sim 150{ }^{\circ} \mathrm{C}$ ); ${ }^{1} \mathrm{H}$ NMR $\left(300 \mathrm{MHz} \mathrm{CDCl}_{3}\right) \delta$ : 8.30 (d, $J=9 \mathrm{~Hz}, 2 \mathrm{H}), 7.84(\mathrm{~d}, J=9 \mathrm{~Hz}, 2 \mathrm{H}) ;{ }^{13} \mathrm{C} \mathrm{NMR}$ $\left(75 \mathrm{MHz}, \mathrm{CDCl}_{3}, 298 \mathrm{~K}\right) \delta: 149.0,132.5,123.3,117.5$, 115.7.

肉桂腈 ${ }^{[11,30]}$ : 无色液体. ${ }^{1} \mathrm{H}$ NMR $\left(300 \mathrm{MHz}, \mathrm{CDCl}_{3}\right)$ $\delta: 7.28 \sim 7.19(\mathrm{~m}, 5 \mathrm{H}), 7.13(\mathrm{~d}, J=15 \mathrm{~Hz}, 1 \mathrm{H}), 5.68(\mathrm{~d}$, $J=18 \mathrm{~Hz}, 1 \mathrm{H}) ;{ }^{13} \mathrm{C} \mathrm{NMR}\left(75 \mathrm{MHz}, \mathrm{CDCl}_{3}, 298 \mathrm{~K}\right) \delta$ : $150.5,133.4,131.2,129.1,127.5,118.3,96.4$.

苯乙腈 ${ }^{[11,30]}$ : 无色液体. ${ }^{1} \mathrm{H}$ NMR $\left(300 \mathrm{MHz}, \mathrm{CDCl}_{3}\right)$ $\delta: 7.21 \sim 7.08(\mathrm{~m}, 4 \mathrm{H}), 3.49\left(\mathrm{~s}, \mathrm{CH}_{2}, 2 \mathrm{H}\right) ;{ }^{13} \mathrm{C}$ NMR $(75$ $\left.\mathrm{MHz}, \mathrm{CDCl}_{3}, 298 \mathrm{~K}\right) \delta: 130.1,129.2,128.03,127.97$, $118.1,23.5$.

辅助材料(Supporting Information) 催化产物腈的 ${ }^{1} \mathrm{H}$ $\mathrm{NMR}$ 和 ${ }^{13} \mathrm{C}$ NMR 谱图. 这些材料可以免费从本刊网站 (http://sioc-journal.cn/)上下载.

\section{References}

[1] Mowry, D. T. Chem. Rev. 1948, 42, 189.

[2] Kukushkin, V. Y.; Pombeiro, J. L. Chem. Rev. 2002, 102, 1771.

[3] Gaspar, B.; Carreira, E. M. Angew. Chem., Int. Ed. 2007, 46, 4519.

[4] Kent, R. E.; McElvain, S. M. Org. Synth. 1945, 25, 61.

[5] Krynitsky, J. A.; Carhart, H. W. Org. Synth. Coll. 1963, IV, 436.

[6] Reisner, D. B.; Horning, E. C. Org. Synth. Coll. 1963, IV, 144.

[7] Kuwano, R.; Takahashi, M.; Ito, Y. Tetrahedron Lett. 1998, 39, 1017.

[8] Das, S.; Zhou, S.; Addis, D.; Enthaler, S.; Junge, K.; Beller, M. Top. Catal. 2010, 53, 979.

[9] Park, S.; Brookhart, M. J. Am. Chem. Soc. 2012, 134, 640.

[10] Pisiewicz, S.; Junge, K.; Beller, M. Eur. J. Inorg. Chem. 2014, 2345 . 
[11] Ren, S.; Wang, Y.; Yang, F.; Sun, H.; Li, X. Catal. Commun. 2019, 120,72 .

[12] Bose, D. S.; Jayalakshmi, B.; Goud, P. R. Synthesis 1999, 1724.

[13] Manjula, K.; Pasha, M. A. Synth. Commun. 2007, 37, 1545.

[14] Enthaler, S.; Weidauer, M. Catal. Lett. 2011, 141, 1079.

[15] Enthaler, S. Eur. J. Org. Chem. 2011, 2011, 4760.

[16] Elangovan, S.; Quintero-Duque, S.; Dorcet, V.; Roisnel, T.; Norel, L.; Darcel, C.; Sortais, J.-B. Organometallics 2015, 34, 4521.

[17] Bezier, D.; Venkanna, G. T.; Sortais, J.-B.; Darcel, C. ChemCatChem 2011, 3, 1747.

[18] Xue, B.; Sun, H.; Wang, Y.; Zheng, T., Li, X.; Fuhr, O.; Fenske, D. Catal. Commun. 2016, 86, 148.

[19] Wang, Y.; Ren, S.; Zhang, W.; Xue, B.; Qi, X.; Sun, H.; Li, X.; Fuhr, O.; Fenske, D. Catal. Commun. 2018, 115, 1.

[20] Luan, H. X.; Sun, H. J.; Xue, B. J.; Li, X. Y. Chin. J. Org. Chem. 2017, 37, 1392 (in Chinese).

(奕化金金, 孙宏建, 薛本静, 李晓燕, 有机化学, 2017, 37, 1392.)

[21] Zhao, H.; Sun, H. J.; Wang, L.; Li, X. Y. Acta Chim. Sinica 2015, 73, 1307 (in Chinese). (赵华, 孙宏建, 王麟, 李晓燕, 化学学报, 2015, 73, 1307.)

[22] Lu, F. G.; Sun, H. J.; Li, X. Y. Chin. J. Chem. 2013, 31, 927.
[23] Qi, X.; Sun, H.; Li, X.; Fuhr, O.; Fenske, D. Dalton Trans. 2018, 47, 2581.

[24] Qi, X.; Zheng, T.; Zhou, J.; Dong, Y.; Zuo, X.; Li, X.; Sun, H.; Fuhr, O.; Fenske, D. Organometallics 2019, 38, 268.

[25] Wang, Z.; Li, X.; Sun, H.; Fuhr, O.; Fenske, D. Organometallics 2018, 37, 539 .

[26] Wang, L.; Sun, H.; Li, X. Organometallics 2015, 34, 5175.

[27] Zhou, H.; Sun, H.; Zhang, S.; Li, X. Organometallics 2015, 34, 1479.

[28] Liu, R. Y.; Bae, M.; Buchwald, S. L. J. Am. Chem. Soc. 2018, 140, 1627.

[29] Wang, Y. Y.; Fu, L. Y.; Qi, H. M.; Chen, S. W.; Li, Y. H. Asian J. Org. Chem. 2018, 7, 367.

[30] Supsana, P.; Liaskopoulos, T.; Tsoungas, P. G.; Varvounis, G. Synlett 2007, 2671.

[31] Ramanathan, A.; Jimenea, L. S. Synthesis 2010, 217.

[32] Ushijima, S.; Moriyama, K.; Togo, H. Tetrahedron 2011, 67, 958.

[33] Kijima, M.; Nambu, Y.; Endo, T.; Okawara, M. J. Org. Chem. 1984, 49, 1434.

[34] Silverman, M.; Bogert, M. T. J. Org. Chem. 1946, 11, 34.

(Zhao, C.) 